\title{
Currículo y prácticas universitarias de vinculación curricular
}

\section{Curriculum and university curricular linking practices}

\author{
María Raquel Coscarelli \\ https://orcid.org/0000-0002-8539-2538 \\ mraquelct@yahoo.com.ar \\ Universidad Nacional de La Plata | Argentina
}

\section{RESUMEN}

Los cruces de disciplinas y saberes provenientes del campo territorial se consideran fuentes curriculares inagotables, fabuloso potencial para la transformación, en un sentido de creciente inclusión social igualitaria y democrática. Las prácticas de vinculación territorial son vistas como parte del campo curricular crítico social.

El artículo retoma algunas investigaciones previas en clave curricular y de formación, y aborda el sentido de núcleos precurriculares emergentes de dichas prácticas. Desde la perspectiva pedagógica, no excluyente de otros posibles ángulos de lectura, la denominada curricularización conlleva a la cuestión de la institucionalidad curricular y los aportes solidarios de la Educación Popular y las perspectivas críticas en la construcción de estas prácticas de enlace universidad-sociedad.

\section{ABSTRACT}

The crossings of disciplines and knowledge from the territorial field are considered inexhaustible curricular sources, fabulous potential for transformation, in a sense of growing egalitarian and democratic social inclusion. Territorial linking practices are seen as part of the social critical curriculum field. The article takes up some previous research in curriculum and training, and addresses the sense of emerging precurricular nuclei of these practices. From the pedagogical perspective, not excluding other possible reading angles, the so-called curricularization leads to the issue of curricular institutionality and the solidarity contributions of Popular Education and the critical perspectives in the construction of these university-society liaison practices.

PALABRAS CLAVE

Currículo, Prácticas de Vinculación Curricular Núcleos de saberes precurriculares.

\section{KEY WORDS}

Curriculum, Curriculum Linking Practices,

Precurricular Knowledge Cores. 


\section{INTRODUCCIÓN: CAMBIOS CURRICULARES}

Los diversos cambios culturales, políticos, epistemológicos, tecnológicos de la sociedad inciden en los procesos de generación del saber, su transmisión, transformaciones y caducidades. Este ciclo cultural, está inserto en las prácticas formativas y en el currículo universitario que las orienta. Los procesos y los sujetos involucrados, "sujetos curriculares" (de Alba, 1995) entraman sus vínculos y desvínculos en las distintas manifestaciones curriculares. La expansión disciplinaria, la dilución de los límites entre las disciplinas y a la vez la aparición de nuevas articulaciones entre saberes de carácter interdisciplinario y en particular, en nuestro interés, los que surgen de la vinculación con las demandas sociales intervienen en la estructuración y dinámica de los currículos contemporáneos.

\section{La expansión disciplinaria, la dilución de los límites entre las disciplinas y a la vez la aparición de nuevas articulaciones entre saberes de carácter interdisciplinario y en particular, en nuestro interés, los que surgen de la vinculación con las demandas socia- les intervienen en la estructuración y dinámica de los currículos contemporáneos.}

Los contenidos, las teorías, la complejidad de los temas, las bibliografías están produciendo modificaciones con una asombrosa velocidad en todos los campos disciplinares transformando los currículos universitarios (Rama 2009). Estas vertientes impactan en el currículo cuya dinámica no siempre es propicia para su incorporación. La disponibilidad para la transmisión y apropiación de los conocimientos emergentes de la especialización disciplinar, la interdisciplinariedad y la vinculación social remiten a cuestiones también complejas con lógicas que dependen de los campos disciplinares y experienciales, sujetos, contextos, poderes y jerarquías instaladas y atribuidas.

Los saberes que provienen del encuentro entre las disciplinas académicas y los múltiples ámbitos sociales están poco explorados. Como admite Bronckart "existen diversos lugares de producción de saberes dotados de historias, de funciones sociales, de estructuras y modalidades de funcionamiento específicos" (2007: 115). Los diversos campos de producción de saberes y sus tipos de discurso (narrativos, teóricos, 
interactivos) pueden variar en extensión, importancia, valor simbólico y legitimidad.

Así también, las innovaciones curriculares, cambios planificados, condensan, en diferentes articulaciones "cuestiones provenientes de las culturas académicas, las situaciones de contexto, la dinámica propia de los campos disciplinarios, el impacto de las políticas de Estado y seguramente otras condiciones que es de imprescindible necesidad identificar y comprender" (Fernández Lamarra, 2015: 12). Estas disímiles producciones pueden ser acordes o contradictorias entre sí, pero todas son fuentes de bienes y saberes culturales.

Estudiar el curriculum nos coloca en una infinitud de perspectivas posibles no sólo de lugares de producción y propagación de conocimientos, sino de los diversos procesos y trayectos de carácter multidireccional, plenos de transacciones reproductivas y creativas, y en ellos las prácticas de vinculación territorial.

\section{Estudiar el curriculum nos coloca en una infinitud de perspectivas posibles no sólo de lugares de producción y propagación de co- nocimientos, sino de los diversos procesos y trayectos de carácter multidireccional, plenos de transacciones reproductivas y creati- vas, y en ellos las prácticas de vinculación territorial.}

\section{PRÁCTICAS DE VINCULACIÓN TERRITORIAL}

Abordar las prácticas universitarias de vinculación territorial y/ o en territorio abre un complejo abanico de cuestiones epistemológicas, culturales, pedagógicas y sobre todo sociopolíticas.

Entendemos como prácticas de vinculación territorial: los trabajos de campo, trabajos integradores -de grado y posgrado-, prácticas profesionalizantes y las que surgen de la función de Extensión Universitaria (EU). Consideramos el concepto de vinculación territorial como abarcativo del tratamiento conceptual de la problemáticas territoriales en las distintas asignaturas, junto con las prácticas en territorio. Este diverso espectro relacional sabemos que requiere singularizar enfoques y características identificatorias que no abordaremos en este trabajo. La tarea, más que compleja, conlleva múltiples interrogantes, entre otros, acerca de cuáles son sus finalidades prioritarias, la transformación que persiguen, los sujetos, prácticas sociales y saberes involucrados. Preferimos esta denominación de prácticas universitarias de vinculación territorial, entendiendo a todas sus manifestaciones como parte de la formación universitaria.

En ellas se expresan, los saberes curriculares y desde nuestra perspectiva destacamos, entre ellos, especialmente, el campo de conformación curricular crítico-social. Este campo, siguiendo a de Alba refiere a un conjunto de conocimientos en sentido amplio, que provee sentido social a disciplinas y profesiones y ubica al universitario como 
sujeto partícipe de la realidad histórico-social en la que se desenvuelve. Las prácticas de vinculación territorial, como todas las prácticas curriculares, están atravesadas por concepciones y acciones que explícita o implícitamente expresan valores particulares y relativos entre sí, aportes teóricos y experienciales que las guían y centralmente cuestiones de formación de sujetos -alumnos, formadores en general y comunitarios-, etc. Esta matriz formativa se articula no siempre en sintonía en el currículo universitario cuya configuración responde a tradiciones institucionales y diversas perspectivas pedagógicas y sociales. En este trabajo se recogen algunos planteos surgidos de investigaciones antecedentes que se retoman en estudios actuales de la autora. Propone el concepto de núcleos precurriculares con la intención de reconocer y configurar saberes que emergen de las prácticas en territorio, es decir previos a su entidad formalizada en alguna instancia del movimiento curricular. Estos saberes emergentes tensan los sentidos del currículo, sus alcances, derivas y los propios procesos formativos inscriptos en ellas.

\section{Las prácticas de vinculación territorial, como todas las prácticas curriculares, están atravesadas por concepciones y acciones que explícita o implícitamente expresan valores particulares y relati- vos entre sí, aportes teóricos y experienciales que las guían}

Con referencia a lo territorial, territorio es un concepto cada vez más utilizado en geografía y otras ciencias sociales a partir de los años 1960 y 1970. El uso corriente del término refería "al espacio de la soberanía o la jurisdicción de un país o sus unidades administrativas, y era especialmente relevante en geografía política" (Capel, 2016:1). Fue adquiriendo nuevos sentidos al ritmo de las transformaciones sociales y de las propias disciplinas científicas "cada vez más de contenido social, pasó a concebirse como espacio social y espacio vivido". Se trata de un referente empírico y también teórico que explica y describe el desenvolvimiento espacial de las relaciones sociales que establecen los seres humanos en los ámbitos cultural, social, político o económico. “El territorio se propuso, así como un eje a partir del cual podrían plantearse adecuadamente ciertos problemas y estimular la colaboración interdisciplinaria" (Op cit). Partimos de esta amplia concepción que excede una mera localización espacial y condensa en él las relaciones históricas político-económicas y socioculturales que lo connotan. Tal como lo entiende Ceceña (2002) el territorio expresa y anida estrechas tramas de saber, relaciones sociales y poder, dominaciones, conflictos, hegemonías y subalternidades. Se trata de un espacio material y simbólico en el que se recrea la historia y la cultura, se construyen utopías y alternativas societales y allí ubicamos los intercambios y significaciones que entretejen las unidades académicas y los ámbitos con los que interactúan. Esta red de relaciones complejas a la luz de los enfoques precedentes obra de sostén y expresión del espacio vivido. En ella se destaca, el currículo al que diversos autores críticos como Kemmis ${ }^{1}$ (1988), de Alba y otros consideran como el pro-

\footnotetext{
${ }^{1}$ Kemmis, S. estudioso del curriculum de la Universidad de Deakin Australia.
} 
yecto educativo y su realización en el aula, y agregamos en espacios con distinto grado de formalidad (Sirvent, 2007), extendiendo el significado a su condición de vínculo entre educación y sociedad.

\section{NÚCLEOS DE SABERES PRECURRICULARES}

El movimiento curricular despliega una compleja trayectoria de generación, transmisión y recreación de saberes, mediados por el poder que se expresa en las diversas intensidades de intereses y luchas de sujetos y fuerzas sociales. Ampliamos en el presente el interés por la EU y otras prácticas curriculares que localizan y problematizan la relación universidad-sociedad y pretendemos caracterizar diversos núcleos curriculares emergentes, como adelantáramos, previos a su entidad formalizada en alguna instancia del movimiento curricular.

Los estudios antecedentes provienen de indagaciones previas de la autora, alrededor de la EU y en particular del concepto de protocurriculum. Incorpora aportes de la pedagoga mexicana Alicia de Alba (2009), en lo que refiere a los contornos sociales y también la categoría núcleos emergentes inspirada en los escritos de Fernando Ulloa ${ }^{2}$ (1969) en los que encontramos interesantes vetas a profundizar.

Denominamos protocurriculum, a ciertas tendencias teórico-prácticas de formación de sujetos que se manifestaban en los proyectos de EU. El concepto parte de la raíz griega protos, lo primero, principal y profundizamos su interpretación en el marco de distintas líneas analíticas que, de manera explícita o implícita, lo aluden en tanto propuesta formativa, aunque ésta no sea su intencionalidad principal ni explícita. El concepto en aquella oportunidad permitió fundamentar dicha faz formativa de la EU pero además comprender a la misma dentro de un continuum que expresa a la Universidad toda y en particular refleja, en sintonía y/o contradicción el sentido de las prácticas formativas institucionales en su currículo real. En esta línea, en los proyectos de extensión -y podríamos ampliar a la mayoría de los proyectos sociales- existe una faceta educativa y en muchos de ellos se enuncia el propósito explícito de formar, estudiantes, miembros de la comunidad, animadores, agentes multiplicadores, replicadores, etc. Un aspecto sustancial que con variada explicitación y profundidad constituye una suerte de "currículo" destinado a la formación de sujetos sociales. La categoría protocurriculum fue permitiendo enriquecer el análisis de los proyectos desde una perspectiva pedagógica.

Fuimos encontrando paulatinamente que había otros autores que, en otros contextos y perspectivas, formulaban conceptos que podían ser asociados al nuestro. Siempre manteniendo esta vinculación con la formación que expresa el currículo, tratando con diferentes niveles de profundidad qué enseñar -contenidos-y a quiénes -sujetos ${ }^{3}-$, pero anclando el sentido de proto en distintos puntos. Así fuimos ampliando sus alcances desde trabajos de Giroux (1998), Achilli (1996), Sirvent (2007) y otros, desplegando cuestiones referidas a la normatividad que conllevan y a su vinculación con el cuerpo teórico de la denomi-

2 La tradición de los estudios institucionales en Argentina tiene entre sus referentes la obra de Ulloa (1965).

3 Da Silva (2001) desarrolla esta doble referencialidad del currículo. Sostiene que comúnmente aludimos al currículo en términos de qué contenidos éste transmite o pone a disposición de los sujetos, pero que no debemos olvidar que dicha pregunta conlleva otra relativa a qué ellos/ellas el currículo pretende formar. 
nada Pedagogía Social y a la Educación Popular.

En Ulloa encontramos el concepto de núcleos preinstitucionales, emergentes surgidos en una comunidad y que constituyen proyectos afines o semejantes. Estos núcleos unen sus intenciones en un proyecto común y tienden a mediatizar su integración, a través de la coexistencia témporo-espacial y la aceptación de un régimen de normas que crea un común denominador, base de su institucionalización. Estos núcleos están representados, en general, por personas (socios fundadores) o por agrupaciones que tienden a fusionarse.

En esa unión, explica Ulloa, participan elementos racionales de beneficio común, y elementos de naturaleza emocional con diferentes contenidos y distinto grado de concienciación. El autor aclara que no plantea la existencia de una fuerza integradora en sí, sino que esta integración constituye de por sí la fuerza ${ }^{4}$. Pero así como dichos núcleos preinstitucionales se unen en un proyecto único, serán también, posteriormente, el origen de la tendencia opuesta, o sea una tendencia a la dispersión. Tomando como fuente al autor, Fernández da cuenta del concepto de núcleos preinstitucionales afirmando que "en toda institución se observa una tendencia a la integración orgánica que se da de inicio entre lo que podríamos llamar los diferentes núcleos preinstitucionales" (1998: 180). Desde el interés de este trabajo resulta importante el registro de los aportes institucionalistas, relevando la existencia de núcleos emergentes de la vida social con potencialidad para crear un proyecto común en nuestro caso de carácter curricular, que a la vez llevan en germen su propia transformación y aún destrucción. Partimos de la idea de que las múltiples institucionalizaciones conllevan culturas y su propagación transmisiva y formativa, por ende, contiene emergentes curriculares, obviamente con distinta intensidad de concientización y de sedimentación.

En lo que respecta a de Alba, el currículo, como dispositivo educativo, en el marco contextual actual, procesa tensiones, resignificadas en la especificidad del campo

"al mismo tiempo que se afirma y reafirma como un dispositivo intrínseco al sistema, [...] se desestructura, se erosiona e irrumpe la voluntad de poder, de saber y de ser en los contextos sociales con aún una fuerza no visible -diríase una fuerza invisible- para una gran parte de los grupos y sectores sociales" (de Alba, 2012: 13).

Si bien el currículo como dispositivo de poder-saber, a su entender, no encuentra en nuestros pueblos proyectos nacionales nítidos, en el marco de la profunda tensión, globalización y crisis estructural generalizada (CEG), aflora en simultáneo la conformación de plurales contornos sociales, de horizontes de futuro, de figuras del mundo (Villoro, 1993). El mundo está en permanentes y profundos movimientos que trastocan sus cimientos y capas constitutivas, dando lugar a nuevas estructuraciones, por medio de luchas, acuerdos, desacuerdos, resis- 
tencias y esperanzas que demuestran la constancia de cambios con rasgos complejos, diversos y versátiles. Un curriculum entreteje relaciones que lo abren en múltiples vías y la sobredeterminación del proyecto -como aclara de Alba en la actualidad, de los contornos sociales, horizontes utópicos o figuras del mundo- se produce en distintos ámbitos culturales y político-sociales.

\section{Un curricullum entreteje relaciones que lo abren en múltiples vías y la sobredeterminación del proyecto -como aclara de Alba en la actualidad, de los contornos sociales, horizontes utópicos o figuras del mundo- se produce en distintos ámbitos cullturalles y político-sociales.}

La doble dinámica del currículo: su reafirmación como dispositivo del sistema que a la vez que se desestructura, expone su voluntad de ser, poder y saber, generando nuevos elementos en los contextos sociales es un sino de época. Estas emergencias conforman los significantes flotantes y vacíos, tendencias que pugnan por nuevas condiciones de vida configurando horizontes utópicos, contornos sociales, y entre otras formaciones y denominaciones, proyectos, figuras del mundo-mundos mejores $^{5}$. Los contornos en el contexto de la CEG expresan nuevas figuras del mundo, de visibilidad poco notoria. Los significantes flotantes pueden mostrar rasgos o elementos de tres tipos: disruptivos, nuevos, provenientes de otras estructuras (de Alba, 2007: 106-108).

Los rasgos disruptivos, según de Alba, pueden ser elementos nuevos e inéditos o los que prevalecen de formaciones anteriores y se activan, se muestran de manera significativa en el espacio social y efectúan dos funciones: contribuir a la dislocación de un orden de cosas y a la vez mostrar indicios de elementos que tienden a conformar los contornos de una nueva configuración discursiva. Obsérvese cierta similitud en un plano más amplio con el enfoque de Ulloa y Fernández respecto de los núcleos preinstitucionales.

Las múltiples direcciones que pueden adoptar estos emergentes, por ejemplo, articular sus elementos del pasado de manera creativa y comprometida a nuevos elementos, o bien el movimiento contrario: reforzar las estructuras existentes o viejas en procesos de restructuración.

Es decir, los elementos inéditos y por ello nuevos pueden emerger como rasgos disruptivos o no y pueden ser resultado de procesos igualmente innovadores. Estos aportes resultan de sumo interés para cualificar los complejos horizontes que las prácticas de vinculación Universidad-territorios van anunciando y delineando.

El análisis reflexivo de estas tres producciones constituye un eje de interés respecto de la exploración de los que denominamos núcleos curriculares emergentes. Nos motiva especialmente la búsqueda de manifestaciones de tendencias sociales que residen en la exterioridad y en la periferia del sistema, como lugares socioculturales desde los que construir el conocimiento. Esta ampliación del enfoque curricular a los espacios de intercambio universidad y sociedad conlleva ubicar-

\footnotetext{
${ }^{4}$ Desde una lectura psicoanalítica encuentra semejanza en la identificación de esta tendencia integradora con el instinto de vida (sobre todo, aclara, en un nivel de organización biológica).

5 Orozco Fuentes (2000:164) basada en de Alba y en el marco del Análisis del Discurso Educativo expresa que los aspectos y fenómenos sociales emergentes y elementos nuevos de significación ponen en crisis y plantean nuevos pro-
} blemas y retos. Estos rasgos pueden constituirse en nuevos contornos de posibilidad de gestar un significado distinto. 
los como territorios de producción de saberes e interrogarse por sus procedencias, sujetos, demandas e intencionalidades a las que refieren. No se trata solo de describir ni recopilar saberes sino de entablar una conversación de reconocimiento de escenarios alternativos del conocimiento social y su incorporación curricular crítico reflexiva con horizonte político transformador.

\section{NORMATIZACIÓN, NÚCLEOS PRECURRICULARES Y CURRÍCULO}

Reconocer el potencial de las actividades de vinculación territorial, por cuanto despliegan formación en términos de sujetos y colectivos en variados sentidos, e intensidades nos ha llevado a profundizar en cuestiones de carácter pedagógico en general y curricular en especial.

En este cometido surgen multiplicidad de interrogantes ya planteados y otros renovados. Entre ellos ¿qué significados atribuir a esta "curricularización" de las acciones? Acordamos con Krotsch (2005) para quien la cuestión social de la Universidad no puede ser planteada en términos de mero extensionismo. Las problemáticas acuciantes del mundo actual deben ser incorporadas al curriculum universitario, a las prácticas de enseñanza, extensión e investigación. En igual sentido de formación antes denominado, crítico social.

\section{Las problemáticas acuciantes del mundo actual deben ser incor- poradas al curriculum universitario, a las prácticas de enseñanza, extensión e investigación. En igual sentido de formación antes denominado, crítico social.}

No se trata solo de reconocer y pugnar por su inclusión en el currículo formal, hecho de enorme valor, esclarecimiento y posicionamiento institucional. Consideramos que a la vez requiere interrogarse en qué perspectiva y tradiciones educativas ubicamos estas prácticas y en este marco problematizar el carácter de la pedagogía que conllevan.

Respecto del carácter de la Pedagogía, no puede ser otro que la criticidad de sus fundamentos e intervenciones. Sin ser nuestro cometido principal, la cuestión pedagógica, su campo de estudio y pertinencia para entender en la materia, amerita algunas breves consideraciones. La Pedagogía no restringe su objeto a la educación y al currículo estrictamente formales. Si bien gran parte de las reflexiones acerca de los procesos de formación y subjetivación, se han dedicado tradicionalmente, a los sistemas educativos formales, y con menor énfasis en el pasado, al abordaje de instancias no formales o de distintos grados de formalidad -asociaciones, ámbitos del trabajo, grupos etáreos y otros que constituyen también fuertes modelos de identificación-, en la actualidad priman los planteamientos situacionales e interactivos más abarcativos y relacionales, en general, propios de posturas no tecnicistas.

En trabajos anteriores y a propósito de la concepción de currículo admitíamos que puede éste ser entendido como una norma organi- 
zadora de las prácticas educativas y el protocurriculum tendencias emergentes que tienden a "institucionalizarse". Coincidíamos, con diversos autores y argumentos (Frigerio,1991; Picco, 2014) acerca del carácter abierto del pensamiento, en este caso curricular a los que se agregan hoy los planteos de Ulloa y de Alba consignados en el apartado anterior. El curriculum instituye, creando normas, valores, formas de pensamiento, lenguajes, y como norma, organiza la síntesis selectiva de saberes que propugna. Pero lo hace en tensión entre lo instituido, formalizado y lo instituyente de sus procesos prácticos en los espacios con distintos grados de formalización. Pero es necesario distinguir entre normatividad y normalización. La normalización supone instalar sentidos y formas de pensamiento estabilizadas, la referencia a un centro fijo, propio de las posturas esencialistas. La curricularización de las actividades de vinculación territorial, por tanto, incluyen un componente, aunque no excluyente, de una cierta formalización e institucionalización normativa. Pero a la vez, el reconocimiento y configuración de los núcleos precurriculares, responden a criterios dinámicos que se construyen en el propio proceso de intercambio.

Los debates educativos sobre dónde se gestan los escenarios del saber y hacia dónde marchan las opciones curriculares invocan las transformaciones sociales y tecnológicas, el avance de las ciencias, de las nuevas teorías epistemológicas, de los nuevos movimientos sociales y culturales, las luchas por la defensa de los derechos humanos, el feminismo, el ecologismo. Con especial localización en nuestra América adquieren visibilidad prácticas ancestrales y del presente desconocidas y desvalorizadas en los discursos dominantes que cruzan y están cruzadas por esas otras tendencias.

\section{Los debates educativos sobre dónde se gestan los escenarios del saber y hacia dónde marchan las opciones curriculares in- vocan las transformaciones sociales y tecnológicas, el avance de las ciencias, de las nuevas teorías epistemológicas, de los nuevos movimientos sociales y culturales, las luchas por la de- fensa de los derechos humanos, el feminismo, el ecologismo.}

El desarrollo de experiencias sociales alternativas de Educación Popular, las Ilamadas Educación Decolonial, Pedagogías del Sur y otras proveen valiosos aportes de producción cultural y social que replantean el campo curricular. El pensamiento y la obra del profesor e investigador Jorge Huergo de nuestra Casa de Estudios, es un referente notable en esta línea, bregando por generar experiencias de formación que partan de la lectura de la realidad y de las necesidades sociales. Recordamos también al profesor e investigador Manuel Argumedo, de extensa actuación en prácticas de vinculación social, quien consideraba que el eje social integrador y ordenador de las actividades de extensión, docencia e investigación debería plantearse en la propuesta curricular.

La reconstrucción de experiencias de la praxis pedagógica es una 
vertiente ${ }^{6}$ que convoca al develado de tradiciones ancladas en nuestras raíces. Tal cual expresa de Sousa Santos (2009), es necesario ampliar las fronteras del conocimiento incorporando otras culturas sometidas, condenadas al olvido y juzgadas desde patrones universales, abrirse a lo que conceptualiza como epistemologías del sur. El autor recupera el pensamiento freireano de ruptura y a la vez de apertura a una nueva articulación de sentido.

En esta perspectiva se ha enriquecido el caudal de experiencias y teorizaciones emergentes a la vez que se problematizan y profundizan obras y autores. Sería muy extenso nominar y destacar sus vertientes . La sistematización de estos saberes, no siempre se cumple y resulta un punto nodal para su recuperación y la transformación curricular en el camino de la producción de nuevas epistemologías, desde la experiencia práctica, frente a las formas de conocimiento dominantes.

El amplio caudal de las tendencias alternativas y las de Educación Popular se han abierto paso y lo hacen a contrapelo de las pedagogías triunfantes, que propugnan silencios y despolitizaciones del campo. Sin embargo, las tradiciones pedagógicas "normalizadoras" hegemónicas no están exentas de las marcas históricas de sus contradicciones y de sus ocultamientos respecto de tendencias alternativas. El pensamiento crítico situado en nuestros pueblos americanos ha desenvuelto un amplio caudal de conocimientos que surgen de nuevas formas de conocer con eje en la emancipación.

\section{CONCLUSIONES}

Se trata de encarar una política de reconocimiento del otro por medio de una conversación horizontal con las experiencias de movimientos sociales, pueblos originarios y otras diversidades, culturas tradicionalmente marginadas de la escuela. Construir nuevos saberes apelando a develar las ausencias de registro, comprensión y empoderamiento de sujetos y culturas excluidos de las normatividades centrales y, lejos de toda perspectiva fijista y esencialista, dar la palabra a tradiciones plurales, históricamente localizadas.

La postura crítica se asume como una actitud y no como un corpus definido, como una "teoría crítica" con parámetros pre-establecidos (Cappellacci Guelman,et all, 2018: 37). Coincidimos en que las vertientes de Educación Popular y de las Pedagogías Críticas no se contraponen, contribuyen solidariamente en la lucha por la educación pública en $A L$ y oponen su fuerza y pensamiento ante los embates repositorios neoliberales y neoconservadores.

Desde este marco, corresponde ahondar en las prácticas de vinculación territorial, entendiendo la complejidad de la tarea que sistematice, valide y jerarquice conocimientos pero que, a la vez, no se distancie de los requerimientos sociales. Supone normativas de "textura abierta" flexibilidad en su accionar y alerta permanente con respecto a restricciones burocráticas y clausuras de sentido.

6 Estela Quintar (2018: 20) de larga trayectoria junto con Hugo Zemelman en IPECAL (Instituto Pensamiento y Cultura en A.L.) -menciona autores "icónicos" Fals Borda, Rodolfo Kusch, Arturo Jaureche Enzo Faletto y los que denomina"profetas mayores" José Carlos Mariategui, Simón Rodriguez, José Martí. En el presente reconocemos, entre otros, a Enrique Dussel, , Ramón Torres Carrillo, Oscar Jara, Adriana Puiggrós y muchos más. 
Argumedo, M; Coscarelli, M. y otros (2002). Capítulo "Las estrategias de formación de sujetos en los proyectos de la extensión universitaria en la Universidad Nacional de La Plata". Publicado en Krotsch, P. (organizador). La Universidad Cautiva. Legados, Marcas y Horizontes. La Plata Editorial Al Margen Págs. 295-309.

Bronckart, Jean Paul (2007). “Desarrollo del lenguaje y didáctica de las lenguas". Bs. As: Miño y Dávila.

Capel, Horacio (2016). "Las ciencias sociales y el estudio del territorio" en Biblio3W Revista Bibliográfica de Geografía y Ciencias Sociales, Vol. XXI, núm. 1.149. Barcelona. Universidad de Barcelona. Accesible en: www.ub.edu/geocrit/b3w1149.pdf, consultado el 30 de marzo de 2017.

Ceceña, Ana E. (2002). "América Latina en la geopolítica estadounidense" en Revista THEOMAI / THEOMAI Estudios sobre Sociedad, Naturaleza y DesarroIlo / Society, Nature and Development Studies, número 6 (segundo semestre de 2002). Ed. Universidad Nacional de Quilmes. Accesible en: revista-theomai. unq.edu.ar/numero6/artcecena6.htm, consultada 30 de marzo de 2017.

Coscarelli, María Raquel y Picco, S. (2006). “Formación de Formadores en los Proyectos de Extensión Universitaria: aportes para la reflexión". Revista Escenarios de la Facultad de Trabajo Social UNLP Año 6. № 11. La Plata Espacio Editorial págs.35- 44.

Coscarelli, María Raquel (comp.) (2009). "La extensión universitaria. Sujetos, formación y saberes". La Plata: Ediciones de Periodismo y Comunicación, UNLP. de Alba, Alicia (1995). "Curriculum: crisis, mito y perspectivas". Bs. As. Ed.: Miño y Dávila.

-----(2007). "El curriculum en el contexto de la crisis estructural generalizada”. En A. de Alba, Curriculum-Sociedad. El peso de la incertidumbre, la fuerza de la imaginación México: IISUE.

------(2012). “Pensar la política educativa universitaria desde el espacio del curriculum. El curriculum como dispositivo educativo de poder-saber. Voluntad de poder y voluntad de ser". En The Fourth World Curriculum Studies Conference, Rio de Janeiro, Brasil.

De Sousa Santos, Boaventura (2009). “Una Epistemología del Sur. La reinvención del conocimiento y la emancipación social". Buenos Aires: Siglo XXI Editores, CLACSO www.boaventuradesouzasantos.pt/media/Introducción_BSS.Pdf

Fernández Lamarra, Norberto. (Comp.) Aiello, M.; Álvarez, M.; Fernández, L.; García, P.; Grandoli, M.E.; Ickowicz. M.; Paoloni, P.; Perez Centeno, C. (2015). “La innovación en las Universidades Nacionales. Aspectos endógenos que inciden 
en su surgimiento y desarrollo". Provincia de Buenos Aires, Argentina: Universidad Nacional de Tres de Febrero.

Fernández, Lidia (1996). "Instituciones Educativas". Buenos Aires: Paidós.

-----(1998). “El análisis de lo institucional en la escuela”. Buenos Aires: Paidós.

Frigerio, Graciela. (1991). "Curriculum: norma, intersticios, transposición y textos (capítulo 1)". Frigerio, Graciela (Comp.), Curriculum presente ciencia ausente. Normas, teorías y críticas 1a. ed., Vol. I, pp.17-45. Buenos Aires: Miño y Dávila; FLACSO.

Guelman, Anahí; Cabaluz, Fabián; Salazar, Mónica (Coordinadores) (2018). "Educación popular y pedagogías críticas en América Latina y el Caribe: corrientes emancipatorias para la educación pública del Siglo XXI". 1a ed. Ciudad Autónoma de Buenos Aires: CLACSO Libro digital, PDF Archivo Digital.

Kemmis, Stephen (1988). "El Curriculum: más allá de la teoría de la reproducción. Madrid: Morata".

Krotsch, Pedro (2005). “Entrevista realizada por del Dr. Manuel Argumedo para la Revista Tram(p)as de la comunicación y la cultura, eje temático: Universidad y compromiso social", Facultad de Periodismo y Comunicación Social, UNLP, Año 4, № 35.

Rama, C. (2009). "La universidad latinoamericana en la encrucijada de sus tendencias UNAH - Universidad Nacional Autónoma de Honduras". Tesis de Doctorado. Facultad de Derecho, Universidad de Buenos Aires, Argentina.

Sirvent, María Teresa (2007). "Revisión del concepto de Educación No Formal". Ficha de Cátedra de Educación No Formal. Buenos Aires: Facultad de Filosofía y Letras U.B.A.

Picco, S. (2014). “Concepciones en torno a la normatividad en la didáctica. Un análisis interdisciplinario de obras teóricas didácticas y curriculares en la Argentina entre 1960 y 1990". (En línea). Tesis del Doctorado en Ciencias de la Educación, Facultad de Humanidades y Ciencias de la Educación, Universidad Nacional de La Plata, publicada en el repositorio institucional de la UNLP"SEDI$\mathrm{Cl}^{\prime \prime}$. Recuperado de http://sedici.unlp.edu.ar/handle/10915/35590

Ulloa, Fernando (1969). “Psicología de las Instituciones. Una aproximación psicoanalítica" en Revista de Psicoanálisis, Vol. XXVI, № 1. Buenos Aires: APA. Introducción consultada en Internet el 30 de marzo de 2017. 